\title{
Conclusions de RARE 2013
}

Intervention d'Emilie Royere (Eurobiomed)

Plus de 300 personnes ont participé à ces rencontres, et plus de 150 rendez-vous d'affaire ont été organisés. Je remercie tous les acteurs qui ont contribué à la réussite de cet événement. Eurobiomed est à vos côtés pour mobiliser la communauté industrielle des PME.

\section{Intervention de Christian Deleuze (Genzyme)}

La dernière table ronde aurait gagné à figurer en début de congrès pour nourrir les discussions des deux journées qui ont suivi. L'engagement des industriels dans le domaine des maladies rares est une action de long terme, collective, menée au quotidien par nos équipes en partenariat avec l'ensemble des acteurs concernés. Du fait des contraintes économiques actuelles, la spécificité du plan national maladies rares, et des efforts financiers et humains qui y sont attachés, doivent être préservés et défendus. Je félicite les organisateurs pour ces deux journées qui ont permis de mettre en valeur l'engagement de chacun des acteurs du monde des maladies rares et d'éclairer le chemin qui nous reste à parcourir.

\section{Intervention d'Odile Kremp (Orphanet)}

Nous avons préféré commencer ces rencontres par l'état actuel du PNMR 2 afin de finir par une table ronde sur l'éventualité d'un PNMR 3. Ces deux journées ont permis d'identifier les avancées et les points critiques des deux premiers plans. En outre, elles ont permis de mieux appréhender la notion de partenariat à différents niveaux. Je remercie tous les membres du comité scientifique qui ont préparé le programme. Je remercie aussi l'équipe d'Eurobiomed qui nous a beaucoup soutenus.

\section{LIENS D'INTÉRÊT}

Les auteurs déclarent n'avoir aucun lien d'intérêt concernant les données publiées dans cet article.

\section{www.medecinesciences.org}

\section{OUI, je m'abonne aux 10 numéros/an de $\mathrm{m} / \mathrm{s}$}

Nom/Prénom :

Société :

Adresse :

$\mathrm{CP}$ : Ville :

E-mail (obligatoire) :

Je joins mon chèque de règlement (libeller votre chèque à l'ordre d'EDP Sciences)

J Je règle par $\mathrm{CB}$ (paiement en ligne sécurisé sur site cf. section « abonnements »)

Je souhaite recevoir une facture

\begin{tabular}{|c|c|c|c|c|}
\hline \multicolumn{2}{|c|}{ Abonnement } & France & UE & $\begin{array}{c}\text { Reste } \\
\text { du monde }\end{array}$ \\
\hline \multirow{2}{*}{$\begin{array}{l}\text { Particuliers } \\
\text { (prix HT) }\end{array}$} & $P+E$ & O $221 €$ & $\bigcirc 300 €$ & $\bigcirc 300 €$ \\
\hline & $E$ & O $152 €$ & O $152 €$ & O $127 €$ \\
\hline \multirow{2}{*}{ Institutions } & $P+E$ & $508 €$ & O $619 €$ & O $642 €$ \\
\hline & $E$ & \multicolumn{3}{|c|}{ sur devis } \\
\hline \multirow{2}{*}{ Étudiants* } & $P+E$ & $\bigcirc 117 €$ & $\bigcirc 165 €$ & $\bigcirc 187 €$ \\
\hline & $E$ & ○ $89 €$ & ) $89 €$ & ○ $75 €$ \\
\hline \multirow{2}{*}{ Enseignants } & $P+E$ & O $146 €$ & O $252 €$ & O $271 €$ \\
\hline & $E$ & O $120 €$ & O $120 €$ & O $101 €$ \\
\hline
\end{tabular}

$P+E$ : Papier et Électronique $\mathrm{E}$ : Électronique

À retourner à : EDP Sciences - Service abonnement
une publication du groupe
17, avenue du Hoggar - P.A. de Courtaboeuf - 91944 Les Ulis Cedex A, France

